\title{
Biological reaction of zirconia ceramic as a new implant material in the dental field
}

\author{
Noriyuki Nagai, Nobuyoshi Takeshita, Junji Hayashi, Yasuhiko Kuwana, \\ Naoki Shirasuga, Haruyoshi Maruyama, Hiromu Sekine* \\ and Youji Fujii** \\ Department of Oral Pathology (Chief: Prof. Noriyuki Nagai), \\ Okayama University Dental School, 2-5-1 Shikatacho, Okayama 700, Japan \\ *Department of Prosthodontics (Chief: Prof. Hiromu Sekine), \\ Tokyo Dental College, 1-2-2 Masago, Chiba 260, Japan \\ ** Research Center of SHINAGAWA Refractories Co., \\ 707 Inbe, Bizen 705, Japan
}

[Accepted for publication: July 15, 1982$]$

Key words: biomaterial / zirconia ceramic/tissue reaction

It has been reported in many articles ${ }^{1-4)}$ that alumina ceramic has excellent tissue affinity and physical properties as a biological implant material. We have also become aware of the fact that zirconia ceramic has excellent physical properties similar to those of alumina ceramic. In this report, the histological affinity of zirconia ceramic has been compared with that of alumina ceramic in rats, and the possibility of using zirconia ceramic as a biological implant material is investigated. This is the first communication for the biological reaction of zirconia ceramic.

\section{Materials and Methods}

Sixteen male Wister rats (body weight, about $200 \mathrm{~g}$ ) were used. After intraabdominal anesthesia, zirconia and alumina ceramic pins (polycrystalline), $1.5 \mathrm{~mm}$ in diameter and $3 \mathrm{~mm}$ in length (produced by SHINAGAWA Refractories Co.), were inserted into both the abdominal subfacial tissue and the mandibular bone. The rats were sacrified 7 , 14,28 , or 56 days after surgical procedure. The specimens were fixed in 10 per cent neutral-buffered formalin, the jaw bones were decalcified, and samples were embedded in paraffin in a routine manner. The paraffin sections were cut at 4 microns and stained with hematoxylin-eosin, Azan, or argyophil stain.

\section{Results}

I. Zirconia Ceramic

(1) In the subfacial tissue

At 7 days after surgical procedure, granular tissue with fibroblastic proliferation and infiltration of some histiocytes or monocytes was observed in the tissue around the zirconia ceramic pins (Fig. 1). The zirconia ceramic pins were closely surrounded by fibrous tissues containing many fibroblasts at 14 days. By 28 to 56 days, the zirconia ceramic pins were completely encapsulated by dense fibrous tissues (Fig. 2).

(2) In the mandibular bone

Prominent proliferation of the fibrous tissue with some inflammatory cell infiltration was observed between the zirconia ceramic pins and the jaw bone tissue at 7 days after insertion. Formation of callus trabeculae was also seen in the regions peripheral to the zirconia ceramic pins (Fig. 3 ). At 14 days after insertion, obvious formation of new bone trabeculae was seen in the peripheral region of the zirconia ceramic pins. By 28 to 56 days, the zirconia ceramic pins were closely surrounded by the new bone trabeculae (Fig. 4). By 56 days, new bone tissue had replaced the mature bone tissue.

II. Alumina Ceramic 


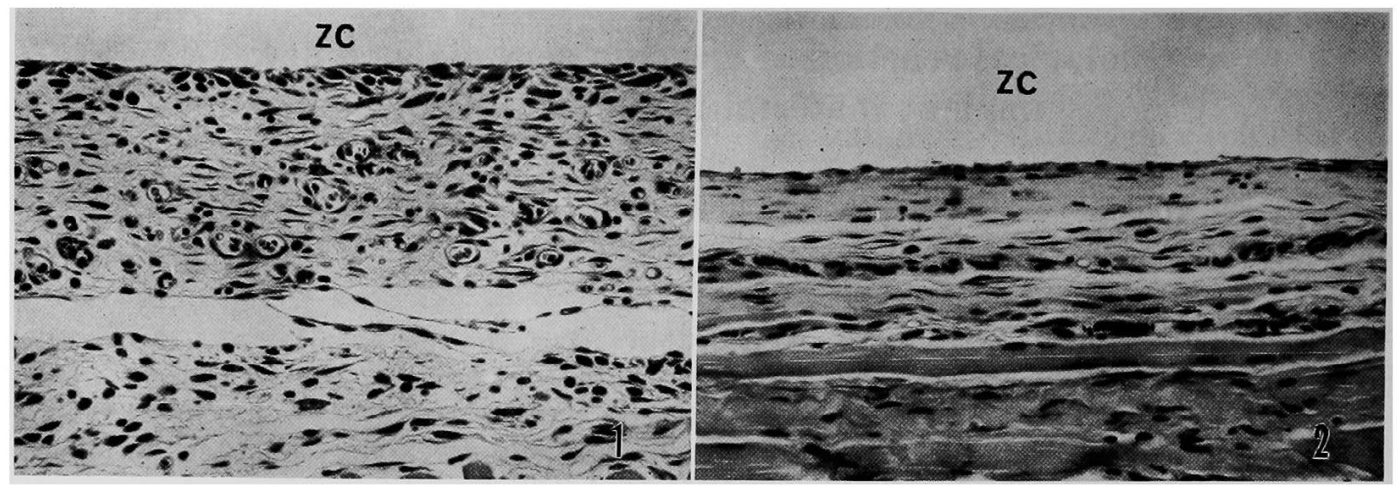

Fig. 1 Fibrous tissue around the zirconia ceramic pin (ZC) in the subfacial tissue at 7 days after insertion. H.E. $\times 190$.

Fig. 2 Prominent fibrosis around the zirconia ceramic pin (ZG) in the subfacial tissue at 28 days after insertion. H.E. $\times 190$.
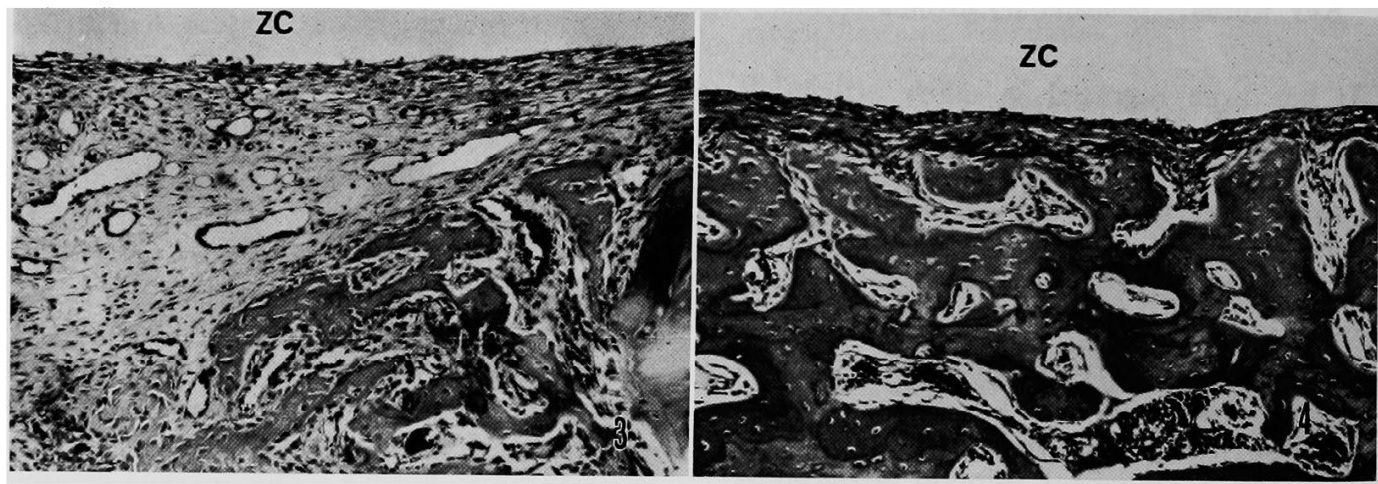

Fig. 3 Active formation of callus in the vicinity of the zirconia ceramic pin ( $Z C$ ) in the mandibular bone at 7 days after insertion. H.E. $\times 95$.

Fig. 4 New bone tissue around the zirconia ceramic pin (ZC) in the mandibular bone at 28 days. Note the mature bone trabeculae close tc the zirconia ceramic pin. H.E. $\times 190$.

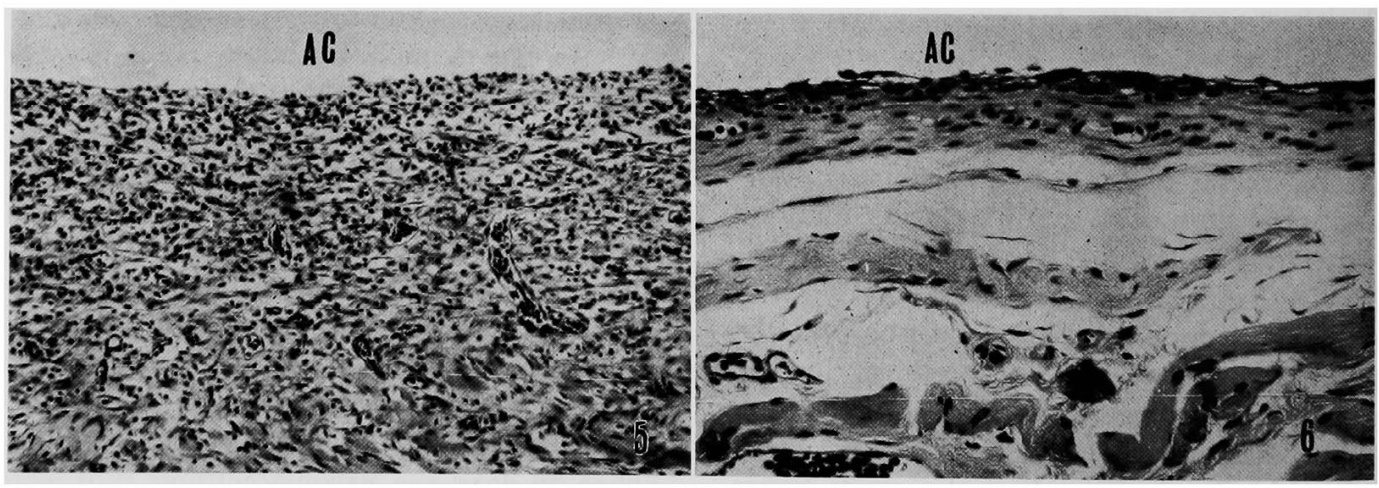

Fig. 5 Moderate infiltration of neutrophils, lymphocytes and histiocytes, and proliferation of fibroblasts around the alumina ceramic pin (AC) in the subfacial tissue at 7 days after insertion. H.E. $\times 95$.

Fig. 6 Prominent fibrosis around the alumina ceramic pin (AC) at 28 days after insertion. H.E. $\times 190$. 


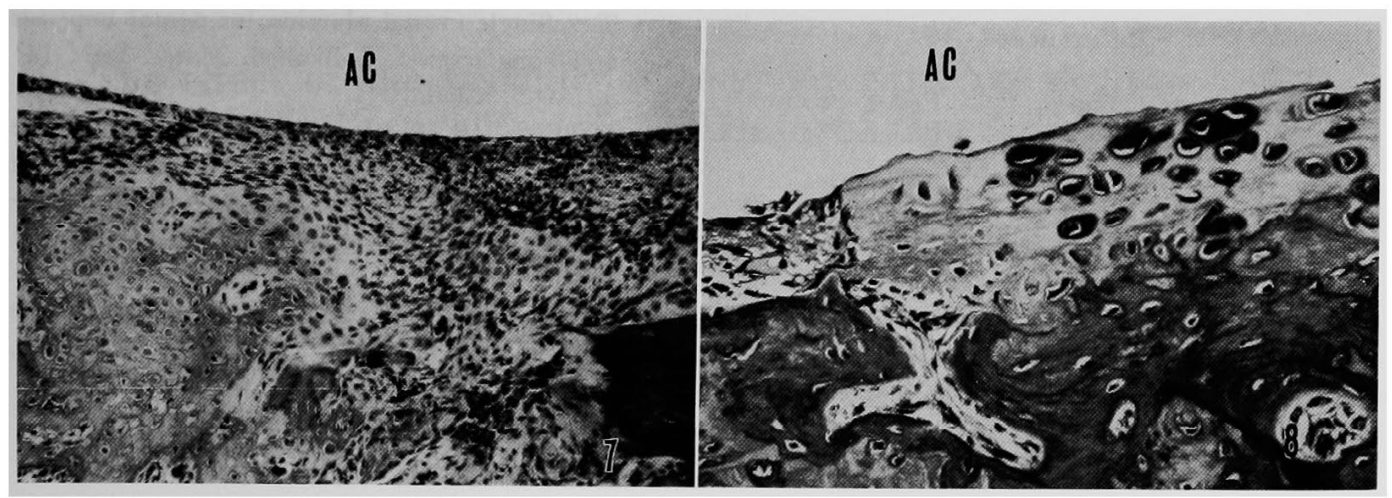

Fig. 7 Note the chondral ossification in the vicinity of the alumina ceramic pin (AC) in the mandibular bone at 7 days after insertion. H.E. $\times 95$.

Fig. 8 Chondro-osseous tissue in touch with the alumina ceramic pin (AC) in the mandibular bone at 28 days after insertion. H.E. $\times 190$.

(1) In the subfacial tissue

By 7 days after surgical procedure, granular tissue containing many fibroblasts and capillaries, had moderately proliferated around the alumina ceramic pins. Some neutrophils, lymphocytes and histiocytes had also infiltrated the vicinity of the alumina ceramic pins (Fig. 5). By 14 days after insertion, the alumina ceramic pins were closely surrounded by fibrous tissue which contained many fibroblasts and a few inflammatory cells. By 28 to 56 days, capsulation of the alumina ceramic pins with dense fibrous tissue was distinct (Fig. 6).

(2) In the mandibular bone

By 7 days after insertion fibroblastic granular tissue with moderate infiltration of lymphocytes, monocytes or histiocytes was observed in the tissue between the alumina ceramic pins and the jaw bone. A relatively severe congestion of capillaries was observed as well. In the region peripheral to the embedded alumina ceramic pins, not only the appositional formation of callus but also chondral ossification was noticeable (Fig. 7). By 14 to 28 days there was a progressive increase in the amount of new bone trabeculae around the alumina ceramic pins through the thin dense fibrous tissues. Chondral ossification, locally touching the ceramic pins, was also observed (Fig. 8). By 56 days after insertion, new bone tissue around the alumina ceramic pins had replaced the mature bone tissues as completely as with the zirconia ceramic pins.

\section{Comments}

It is clear that zirconia ceramic and alumina ceramic have considerable biological affinity: these ceramics were completely encapsulated with dense fibrous tissue in the soft tissue and were closely surrounded by new bone tissue in the jaw bone 28 to 56 days after surgical procedure. However, it was noticeable that the alumina ceramic induced a relatively more prominent inflammatory reaction than zirconia ceramic in the early stages after insertion into the soft tissue, and chondral ossification in the jaw bone. It has been reported that mesenchymal cells differentiate into chondrocytes when they are compressed and cultivated under low oxygen pressure conditions ${ }^{5)}$. It is possible, therefore, that the chondral ossification around the alumina ceramic pins suggest a functional disturbance of the microcirculation caused by congestion or some irritation in the tissue around the alumina ceramic pins. We demonstrated that zirconia ceramic had excellent biological affinity comparable to that of other biomaterials. As zirconia ceramic has most desirable physical properties as well, these results suggest that zirconia ceramic may be a favorable implant material in the dental field. 


\section{References}

1) Harms, J. and Mäusle, E.: Tissue reaction to ceramic implant material. J. Biomed. Mater. Res., 13: 67-87, 1979.

2) Kawahara, H., Yamagami, A., Imanishi, Y. and Nishida, T.: Studies of the bioadaptability of metallic and ceramic implants by means of tissue culture. J. dent. Res., 52 (Suppl. 1004, 1975.

3) Kawahara, H., Hirabayashi, M. and Shikita,
T.: Single crystal alumina for dental implants and bone screws. J. Biomed. Mater. Res., 14: 597-605, 1980.

4) Mckinney, R. U. Jr. and Koth, D. L.: The single crystal sapphire endosteal dental implant material characteristics and 18-month experimental animal trials. J. Prosthet. Dent. 47: 69-84, 1982.

5) Basset, C. A. L.: Current concepts of bone formation. J. Bone. Jt. Surg. 44A: 1217-1244, 1962. 